\title{
Metal Organic Frameworks for Selective Degradation of Amoxicillin in Biomedical Wastes
}

\author{
Marcos V. Paula, ${ }^{a, b}$ Amanda L. Barros, ${ }^{b}$ Kaline A. Wanderley, ${ }^{b}$ Gilberto F. de Sá, ${ }^{b}$ \\ Marcos Eberlin, ${ }^{c}$ Thereza A. Soares ${ }^{*, b}$ and Severino Alves $J r^{*}, b$ \\ aPrograma de Pós-Graduação em Ciência dos Materiais, \\ Universidade Federal de Pernambuco (UFPE), 50590-470 Recife-PE, Brazil \\ ${ }^{b}$ Departamento de Química Fundamental, Universidade Federal de Pernambuco (UFPE), \\ Cidade Universitária, 50740-560 Recife-PE, Brazil
}

\author{
'Laboratório Thomson de Espectrometria de Massas, Instituto de Química, \\ Universidade Estadual de Campinas, 13083-970 Campinas-SP, Brazil
}

\begin{abstract}
The accumulation of antibiotics in wastewater has led to the development and spreading of antibiotic resistance in the environment. Amoxicillin (Amox), a beta-lactamic antibiotic, is one of the most frequently consumed antibiotics in the world. We have applied two metal-organic frameworks (MOFs) containing zinc(II) as platforms to degrade Amox. We have predicted the adsorption of this antibiotic via molecular docking calculations which have been further corroborated by means of Fourier transform infrared and UV-Vis spectroscopies, thermogravimetric analysis, X-ray diffraction and scanning microscopy measurements. We have subsequently performed mass spectrometry analysis of Amox@zeolitic imidazolate framework-8 (ZIF-8) and Amox@Zn(1,4-benzenedicarboxylate) (ZnBDC) to demonstrate the degradation of Amox upon contact with the Zn-containing frameworks. We propose a possible pathway for the degradation of Amox involving the cleavage of the four-membered $\beta$-lactam ring. These $\mathrm{Zn}$-containing frameworks provide a biocompatible platform for the degradation in solution of Amox, which should also be suitable to degrade other $\beta$-lactam antibiotics.
\end{abstract}

Keywords: $\beta$-lactamase activity, gentamicin, $\beta$-lactam catalysis, penicilloic and penilloic acids, biocompatible frameworks

\section{Introduction}

In the last decade, antibiotics have emerged as novel environmental pollutants. The widespread use of antibiotics in human and veterinary medicine, animal husbandry, plant production and aquaculture has led to high consumption and the gradual accumulation of antibiotics in the environment (e.g., wastewater, landfills, industrial and hospital effluents). ${ }^{1-6}$ From 100,000 to 200,000 t of antibiotics are consumed per year in hospitals, homes, veterinary use and aquaculture throughout the world. ${ }^{7}$ On average, high-income countries generate ca. $0.5 \mathrm{~kg}$ of hazardous waste per bed per day whereas low-income countries generate ca. $0.2 \mathrm{~kg}$. Furthermore, low-income countries do not separate hospital waste into hazardous or non-hazardous wastes, making the real quantity of

*e-mail: thereza.soares@ufpe.br; salvesjr@ufpe.br hazardous waste much higher, as reported by the World Health Organization (WHO).

Environmental accumulation of antibiotics has raised serious concerns about the induction of antibiotic resistance ${ }^{8-10}$ The exposure of bacteria to the subinhibitory antibiotic concentrations found in many natural environments such as sewage water and sludge, rivers, lakes and even drinking water is a crucial aspect of the current antibiotic resistance crisis. ${ }^{11-14}$ Furthermore, the incomplete absorption or metabolism of antibiotics in target organisms may lead to excretion rates from 5 to $90 \%$ of the dose in the form of metabolites or as parent compounds..$^{15,16}$ The chemical structure of antibiotics often contains cyclic moieties such as benzene rings, piperazine units, hexahydropyrimidines, sulfonamides, quinolone, and morpholine groups. ${ }^{5}$ Upon metabolic processing in humans and animals, these meta-stable compounds yield activated metabolites, which are continuously released in 
the environment. ${ }^{4,17,18}$ The reactive properties of antibioticderived metabolites are largely unknown, as well as the environmental impact of these compounds. Therefore, there is an urgent demand for the development of novel and efficient techniques to minimize water contamination by antibiotics.

Among the most efficient methods to remove pharmaceuticals in wastewater are carbon-activated adsorption-filtration, ${ }^{19,20}$ Fenton oxidation ${ }^{21,22}$ and photocatalytic degradation. ${ }^{23,24}$ However, these methods have some major drawbacks. The physical process using activated carbon and membrane filtration is limited to the transfer of antibiotics from one phase to another without dealing with its chemical degradation. Advanced oxidation technologies such as Fenton oxidation and photocatalytic degradation are often used for amoxicillin (Amox) degradation. However, a major problem of the process of the Fenton oxidation are the large volumes of iron sludge generated in the process, causing other environmental problems. ${ }^{21,25}$ Photocatalytic degradation has high operational cost and the photocatalytic reactions are generally slow compared to the rates of conventional chemical reactions, making it difficult to treat large volumes of wastewater. ${ }^{26,27}$ Therefore, new technologies are needed that can effectively remove antibiotics from the aquatic environment.

Metal-organic frameworks (MOFs) are infinite crystalline structures constructed by the self-assemblage of metal ions or clusters coordinated to multifunctional organic ligands. Depending on the resulting structure, the MOF skeleton can be flexible, combining a high cavity volume, regular crystallinity, and the presence of tunable organic groups within the framework. ${ }^{28}$ These properties provide more active adsorption sites and reaction centers ${ }^{29}$ required for chemical degradation. Furthermore, MOFs can exhibit high biocompatibility and low toxicity depending on the choice of organic linkers and metal constituents. Several novel MOFs have also been developed for increased water stability. ${ }^{30}$ Some of these frameworks can endure water immersion for long periods of time in $\mathrm{pH}$ ranging from 0 to $12 .{ }^{31}$ Therefore, MOFs have lately emerged as promising candidates for the treatment of wastewater. ${ }^{32}$

Amox is a stable acid, semi-synthetic drug within the penicillin class of $\beta$-lactam antibiotics widely used in both human and veterinary medicine to treat infections caused by Gram-positive or Gram-negative bacteria. ${ }^{33}$ Amox is chemically described as $(2 S, 5 R, 6 R)-6$-[[( $2 R)$-2-amino2-(4-hydroxyphenyl)acetyl]amino]-3,3-dimethyl-7-oxo4-thia-1-azabicyclo[3.2.0]heptane-2-carboxylic acid.

Due to high consumption and continuous discharge, Amox has been detected in environmental samples at concentrations ranging between $\mu \mathrm{g} \mathrm{L}^{-1}-\mathrm{ng} \mathrm{L}^{-1}{ }^{6,34}$ It has been further shown that subinhibitory concentrations of Amox can induce bacterial resistance in Escherichia coli, Listeria monocytogenes, Pseudomonas aeruginosa and Salmonella enterica. ${ }^{9}$ The biorefractory nature of Amox limits its effective removal in urban wastewater treatment plants through conventional treatment processes. ${ }^{35,36}$ Hence, the use of alternative treatment techniques resting upon the degradation of Amox into non-toxic intermediates is highly desirable. Several investigations have been reported in the literature using MOFs as agents for the degradation of Amox. ${ }^{37-39}$

In this report we describe the adsorption and degradation of Amox by the $\mathrm{Zn}$-containing frameworks zeolitic imidazolate framework-8 (ZIF-8) and Zn(1,4-benzenedicarboxylate) (ZnBDC). We have initially performed molecular docking calculations to estimate the binding affinity ${ }^{40}$ of Amox for ZIF-8 and ZnBDC. We have further used gentamicin (Gen) as a negative control since our computational calculations predicted that this antibiotic does not bind to the ZIF-8 and $\mathrm{ZnBDC}$ frameworks. The computationally predicted adsorption of Amox, but not Gen, to ZIF-8 and ZnBDC was confirmed by the means of Fourier transform infrared (FTIR) and UV-Vis spectroscopies, thermogravimetric analysis (TGA), X-ray diffraction (XRD) and scanning electron microscopy (SEM) measurements. We have further performed mass spectrometry (MS) analysis of Amox@ZIF-8 and Amox@ZnBDC to demonstrate the degradation of Amox upon contact with the $\mathrm{Zn}$-containing frameworks. Gen does not undergo any chemical transformation in presence of either ZIF-8 or ZnBDC. We assert that the Zn metal catalyzes the degradation of the beta-lactam ring in Amox, and propose a possible pathway for its degradation involving the cleavage of the four-membered $\beta$-lactam ring. Therefore, these $\mathrm{Zn}$-containing frameworks provide a biocompatible platform for the degradation in solution of $\beta$-lactam antibiotics (e.g., penicillins, cephalosporins, cephamycins, carbapenems and monobactams), the most successful class of antibiotics developed so far. ${ }^{41}$

\section{Experimental}

\section{Experimental procedure}

All the reagents used were analytically pure unless stated otherwise. Solutions were prepared in purified water purified by the Milli-Q gradient Millipak ${ }^{\circledR} 40$ system. Amox ( $\geq 900 \mu \mathrm{g}$ per $\mathrm{mg}$ ) and Gen sulfate salt (ca. $600 \mu \mathrm{g}$ Gen per mg) were supplied by Aldrich. The other chemicals used were $\mathrm{NaOH}$ (Dinâmica), methanol (Dinâmica), $\mathrm{Zn}\left(\mathrm{NO}_{3}\right)_{2} \cdot 6 \mathrm{H}_{2} \mathrm{O}$ (Vetec), benzenedicarboxylic acid 
(Aldrich), KOH (Cinética), methanol (Dinâmica), formic acid (Vetec). ZIF-8 was acquired from Aldrich under the commercial name Basolite ${ }^{\mathrm{TM}} \mathrm{Z1200}$. The material was used without previous treatment. The procedure for the synthesis of the ZnBDC used in this work was developed in our group using previously published procedures. ${ }^{42,43}$ In a typical synthesis $0.056 \mathrm{~g} \mathrm{Na}_{2} \mathrm{BDC}$ and $0.148 \mathrm{~g} \mathrm{Zn}\left(\mathrm{NO}_{3}\right)_{2} \cdot 6 \mathrm{H}_{2} \mathrm{O}$ were dissolved in $4 \mathrm{~mL}$ of water. The reactant mixture was placed in a $10 \mathrm{~mL}$ vessel, sealed and placed in a microwave oven (Discover, CEM) at $120^{\circ} \mathrm{C}$ for $10 \mathrm{~min}$, using a maximum power of $60 \mathrm{~W}$. The solid result was filtered, washed with water and acetone to remove excess reactants, and finally dried in vacuum.

Diffractograms were recorded at room temperature in the range of 5 to $50^{\circ}$ in a Rigaku 2400 DMAX XRD with $\mathrm{Cu} \mathrm{K} \alpha(0.15 \mathrm{~nm})$. TGA was performed in a Shimadzu DTG-60H instrument in the range from room temperature to $800^{\circ} \mathrm{C}$ using an alumina crucible with about $8.0 \mathrm{mg}$ of sample, under dynamic nitrogen atmosphere $\left(50 \mathrm{~mL} \mathrm{~min}{ }^{-1}\right)$ and with a heating rate of $10{ }^{\circ} \mathrm{C} \mathrm{min}^{-1}$. The vibrational spectra in the infrared region (IR) were obtained Bruker IFS $66 \mathrm{v} / \mathrm{S}$ FTIR spectrometer in the range of $4000-400 \mathrm{~cm}^{-1}$. For SEM the samples were prepared on carbon tape on an aluminum support and coated with a 10-20 nm gold film, using a Bal-Tec SCD 050 sputter coater. Images were recorded by an SEM (Jeol JSM-5900) operating at a voltage of $15 \mathrm{kV}$, a 4.0 probe and a working distance of 18 to $24 \mathrm{~nm}$. The electrospray ionization mass spectrometry (ESI(+)-MS) were obtained using Micromass MS, in which samples were diluted in a solution of methanol and water $(1: 1)$ with $0.1 \%$ formic acid and analyzed by direct infusion; the flow used ranged from 10 to $30 \mu \mathrm{L} \mathrm{min}^{-1}$, in positive mode. The spray voltage, temperature, capillary voltage and other parameters were adjusted according to the sample in order to obtain a maximal response, using nitrogen as the drying gas. The acquisition of the MS was carried out $60 \mathrm{~s}$ after insertion. The spectra were acquired in the range $50-1000 \mathrm{~m} / \mathrm{z}$. In the experiments MS/MS, an ion of interest was selected and fragmented. The electronic absorption spectra in the UV and Vis regions of the obtained supernatants were performed for each system in a PerkinElmer Lambda 650 spectrophotometer, using deuterium and halogen lamps, scanning from 200 to $600 \mathrm{~nm}$.

Drug loading was performed under stirring for 1,3 and 7 days of $100 \mathrm{mg}$ of the framework (ZIF-8 or ZnBDC) in $10 \mathrm{~mL}$ aqueous solution containing $300 \mathrm{mg}$ of drug (Amox or Gen) as described by Horcajada et al. ${ }^{44}$ To the solution containing Amox it was added $1 \mathrm{~mL}$ of $\mathrm{KOH}\left(1 \mathrm{~mol} \mathrm{~L}^{-1}\right)$ for the solubilization of the drug. After the incorporation period, the suspensions containing drug and MOF were subjected to the centrifugation process and drying under vacuum.

\section{Computational details}

Atomic coordinates for the ZnBDC and ZIF-8 frameworks were taken from the Cambridge Structural Database (CSD1821299 and CSD602542, respectively). Molecular docking calculations were performed using the AutoDock $4^{45-48}$ software combined with the AutoDock Tools. ${ }^{49}$ Partial charges for the receptor atoms were assigned according to AMBER86 force field parameters ${ }^{50}$ while ligand charges were calculated with the Gasteiger method. ${ }^{51}$ Dihedral angles were treated as fully flexible for ligands. Grid resolution and center were adjusted to each one of the systems. Grid maps of $126 \times 126 \times 126$ points with point spacing of $1 \AA$ were centered at the ZnBDC and ZIF-8 structures. The Lamarckian genetic algorithm (LGA) was used with the following parameters: 150 random individuals in an initial population, a maximum number of 2,500,000 energy evaluations, a maximum number of 27,000 generations with mutation and crossover rates of 0.02 and 0.08 , respectively. An optional elitism parameter equal to 1 was applied, determining the number of top individuals that will survive into the next generation. A maximum of 300 iterations per local search was allowed. The probability of performing a local search on an individual was 0.06 where the maximum number of consecutive successes or failures before doubling or halving the search step was 4. A total of 100 LGA runs were performed. After the conformational search, docked conformations were sorted in the order of increasing energy. The coordinates of the lowest energy conformation were clustered based on a root-mean-squared-deviation (RMSD) of 2.0 $\AA . .^{46-48} \mathrm{~A}$ more detailed description of the methodology employed has been previously presented. ${ }^{52,53}$ Coordinates and trajectories were visualized with the software VMD version 1.9.1. ${ }^{54}$ The atomic coordinates for Amox and Gen were built and the geometry optimized with the software MarvinSketch 5.3.1. ${ }^{55}$

\section{Results and Discussion}

The adsorption or degradation of the drug was first assessed by visual inspection of coloration change during the formation of the complex. Amox in solution has a pallid orange color. Contact with ZnBDC and ZIF-8 during the time period of up to 7 days changes the white color, characteristic of the MOFs, to brown (Figure S1, Supplementary Information (SI) section). Gen in contact with ZnBDC and ZIF-8 did not exhibit any alteration of color during the time period of up to 7 days. These results suggest that Amox is either incorporated into or degraded by the framework, whereas Gen may not have 
been incorporated in the materials. In order to further investigate these findings, the drug-MOF systems were characterized by XRD (Figures S2-S7, SI section), SEM (Figure S8, SI section), TGA (Figures S9-S12, SI section), FTIR spectroscopy (Figures S13-S16, SI section), UV-Vis spectroscopy and MS. The supernatant was analyzed by MS and UV-Vis spectroscopy.

\section{Molecular docking calculations}

Molecular docking calculations were carried out to estimate the binding conformation and binding affinity of Amox and Gen onto the ZnBDC and ZIF-8 frameworks in different media (acidic or basic) conditions. ${ }^{28}$ Drug molecules were docked onto the framework structures and the lowest energy conformer was selected for each of the compounds tested (Figure 1). The lowest energy conformer is defined as the conformation of the ligand with the most favorable interaction energy as given by an empirical energy function ${ }^{48}$ and selected out of ca. $10^{6}$ sampled conformations. ${ }^{47,52,53,56}$ Ideally, the lowest energy conformers should also belong to the most populated conformational cluster. This was the case for Amox based on an RMSD of $1.0 \AA$.
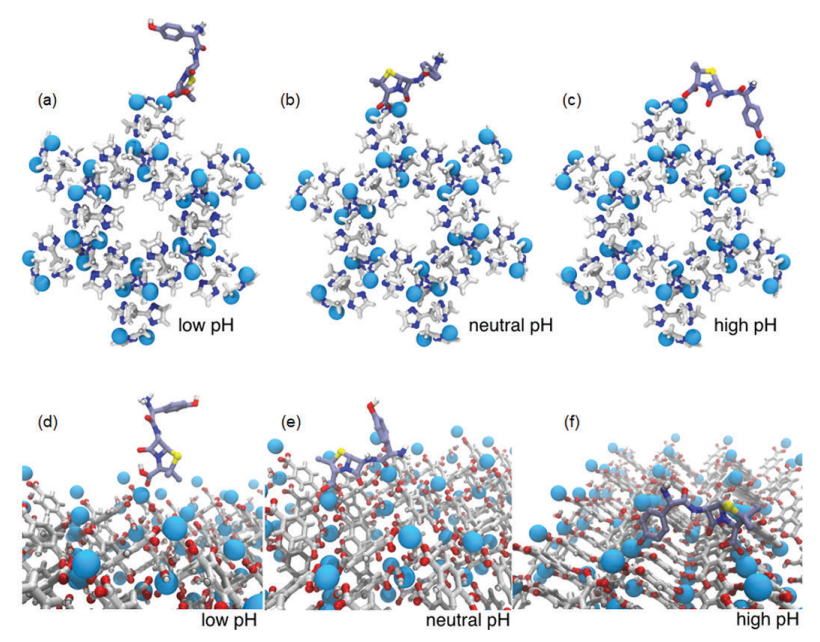

Figure 1. Lowest energy conformers of Amox bound to (a-c) ZIF-8 and (d-f) ZnBDC under different (acidic, neutral or basic) media conditions emulated as different protonation states of the antibiotic. The respective lowest energy conformers are representative of the most populated conformational cluster. A total of $4.05 \times 10^{6}$ conformations were sampled for each one of the conformers.

Amox adsorbs to both frameworks through electrostatic interactions between its carboxylate groups and the $\mathrm{Zn}^{2+}$ metals in the material surface (Figure 1). Depending on the protonation state of Amox, additional interactions with the $\mathrm{Zn}^{2+}$ metal are observed. Amox binds to the ZIF- 8 and $\mathrm{ZnBDC}$ frameworks in a similar fashion under acidic and basic conditions (Figure 1). In basic medium, Amox binds to the framework surface by bridging two different $\mathrm{Zn}^{2+}$ atoms via electrostatic interactions to the thiazolidinic carboxylate and the deprotonated hydroxyl group (Figure 1). In acidic medium, Amox binds to a single $\mathrm{Zn}^{2+}$ atom through to the thiazolidinic carboxylate. In neutral conditions, Amox binds to two $\mathrm{Zn}^{2+}$ atoms via the thiazolidinic carboxylate and the $\beta$-lactam carbonyl groups in the case of ZIF-8, whereas in the $\mathrm{ZnBDC}$ it also binds to two $\mathrm{Zn}^{2+}$ atoms, but this time via the thiazolidinic carboxylate and the carbonyl from the acetoamido group (Figure 1). Based on the MS measurements for both systems (see below), both binding modes at neutral conditions allow the degradation of the antibiotic by the frameworks. The lowest energy Amox conformer in basic medium represents $99 \%$ (ZIF-8) or 98\% (ZnBDC) of the sampled conformations, whereas in neutral medium, it corresponds to $75 \%$ (ZIF-8) and $82 \%$ (ZnBDC), and at acidic medium, 65\% (ZIF-8) and 78\% (ZnBDC). These are merely a theoretical extrapolation since both frameworks are unstable in extreme media conditions. Estimated Amox binding affinity constants from the molecular docking calculations range between $10^{-3}$ to $10^{-4} \mathrm{~mol} \mathrm{~L}^{-1}$ for ZIF-8, and $10^{-7} \mathrm{~mol} \mathrm{~L}^{-1}$ or higher for ZnBDC. Although these values should not be taken with caution due to the simplified potential energy function used in the molecular docking approach, it suggests that Amox adsorbs more strongly to ZnBDC than to ZIF-8.

Molecular docking calculations for Gen onto the ZnBDC and ZIF-8 frameworks did not result in any adsorbed conformation. Estimated affinity constants for this drug had positive values, indicative of unfavorable binding to both frameworks. These findings are consistent with the XRD and TGA measurements as discussed below.

\section{X-Ray powder diffraction}

Powder XRD was performed in order to examine the samples' crystallographic phases qualitatively, as well as to evaluate possible alterations in the MOF structures after being exposed to the drugs. XRD patterns for Amox and Gen are presented in Figures S2 and S3 (SI section). The ZnBDC framework kept the same crystal structure before and after contact with the Gen solution for a time period of up to 7 days (Figure S4, SI section). ${ }^{28,57}$ Conversely, the XRD patterns for ZIF-8 before and after contact with Gen shows alterations (Figure S5, SI section), which may indicate drug adsorption to the framework (see further discussion of the TGA data below). The XRD patterns of the frameworks present thin peaks, indicating high crystallinity and in good agreement with previous studies reported in the literature ${ }^{58,59}$ Conversely, the diffractograms obtained for ZnBDC show significant changes in peak 
positions and relative intensities upon suspension in Amox solution for 1, 3 and 7 days (Figure S6, SI section). The XRD patterns for ZIF-8 show the same crystal structure after contact with Amox solution after 1, 3 and 7 days (Figure S7, SI section), pointing to change in the crystal structure of ZnBDC and ZIF-8 due to reaction with Amox. It has been previously shown by Rietveld refinement that ZnBDC exhibits one single phase before or after suspension in Gen solution, whereas it exhibits two or more crystal phases when suspended in Amox solution. ${ }^{28}$

\section{Scanning electron microscopy}

The morphology of the samples were evaluated qualitatively by SEM (Figure S8, SI section). The micrograph of ZnBDC shows crystals in small and large clusters with well-defined shapes and slightly deformed surfaces. The micrograph of commercial ZIF-8 shows crystalline particles in the nanometric regime, possibly due to fast nucleation during synthesis. ${ }^{60}$ Gen forms spherical particles of ca. $10 \mu \mathrm{m}$, whereas Amox forms spicules, some with large and elongated shapes and irregular length. The findings for Amox are in line with previous studies in the literature. ${ }^{61,62}$

\section{Thermogravimetric analysis}

The stability and thermal decomposition of the samples were evaluated by TGA in the presence and absence of the drugs (Figures S9-S12, SI section). The thermoanalytical profile of $\mathrm{ZnBDC}$ displays two steps. The first, in the region of $150^{\circ} \mathrm{C}$, can be interpreted as the loss of coordination water representing $14 \%$ of the total mass. The next step, between 350 and $550{ }^{\circ} \mathrm{C}$, corresponds to the thermal decomposition of the organic ligand and loss of $56 \%$ of the total mass. Similar results are reported in the literatrure. ${ }^{42}$ The thermoanalytical profile of ZIF-8 displays two events in the range of 350 to $750{ }^{\circ} \mathrm{C}$, which can be interpreted as mass loss of the imidazolate linker, which represents $70 \%$ of the total mass. The remaining mass corresponds to the compound $\mathrm{ZnC}$, yielded after the loss of organic ligand. Similar results were previously reported by Ordoñez et al.$^{60}$ The thermoanalytical profile of Gen shows loss of hydration water at about $100{ }^{\circ} \mathrm{C}$, which corresponds to $10 \%$ of the total mass. The remaining mass corresponds to the decomposition of the Gen skeleton. The thermoanalytical profiles for Gen in the presence of ZnBDC exhibited a similar thermal behavior to that of the pure framework. The TGA profiles for ZIF-8 after suspension in the Gen solution feature loss of mass from both drug and framework (Figures S9-S12, SI section). These data indicate the drug adsorbs to the framework.

The thermoanalytical profile of Amox shows a loss of hydration water at the temperature range of 65 to $130{ }^{\circ} \mathrm{C}$, which corresponds to a loss of $12 \%$ of the total mass. Additionally, three consecutive events are observed: the first event takes place in the range of 160 to $210{ }^{\circ} \mathrm{C}$, with mass loss of $11 \%$; the second event occurs in the interval of 211 to $320{ }^{\circ} \mathrm{C}$, with mass loss of $26 \%$; and the last one is observed in the range of 370 to $700{ }^{\circ} \mathrm{C}$, which corresponds to $51 \%$ of total mass. These events can be interpreted as the decomposition of Amox, leading to the complete degradation of sample. The thermal analysis curve for the mixture Amox and ZnBDC shows four mass loss events whose profile is similar to the pure Amox thermal profile; likewise for the mixture Amox and ZIF-8. The first event is related to the loss of hydration water molecules trapped in the compound. The second event is attributed to the loss of the Amox and their degradation products. The third and fourth decomposition events correspond to the simultaneous thermal decomposition of Amox, their degradation products and ZnBDC, followed by carbonization of the material. In addition, the thermal stability of all samples was analyzed by comparing the 5\% $\left(\mathrm{T}_{5}\right)$ and $10 \%\left(\mathrm{~T}_{10}\right)$ weight loss temperature of the samples (Table S1, SI section). In addition, residual mass at $700{ }^{\circ} \mathrm{C}$ is also reported.

\section{Infrared spectroscopy}

FTIR spectra were obtained for ZnBDC and ZIF-8 before and after suspension in solutions of Gen and Amox for 1, 3 and 7 days (Figures S13-S16, SI section). The spectrum for $\mathrm{ZnBDC}$ exhibits one band centered at $3249 \mathrm{~cm}^{-1}$ attributed to $\mathrm{O}-\mathrm{H}$ stretching due to water coordination. The strong absorption band at $1576 \mathrm{~cm}^{-1}$ is attributed to $\mathrm{C}=\mathrm{O}$ stretching, which is displaced due to coordination with the metallic center. The FTIR spectrum for ZIF-8 exhibits small absorption bands at 3131 and $2923 \mathrm{~cm}^{-1}$ related to the aromatic $\mathrm{C}-\mathrm{H}$ stretching and the aliphatic $\mathrm{C}-\mathrm{H}$ stretching in the imidazole, respectively. The band at $1605 \mathrm{~cm}^{-1}$ corresponds to the $\mathrm{C}=\mathrm{C}$ stretching. The axial deformation of the $\mathrm{C}=\mathrm{N}$ band occurs at $1576 \mathrm{~cm}^{-1}$, and the axial deformation of the $\mathrm{C}-\mathrm{N}$ at $1145 \mathrm{~cm}^{-1}$. The absorption band at $421 \mathrm{~cm}^{-1}$ is associated with the stretching of the coordination $\mathrm{Zn}-\mathrm{N}$. Similar results are reported by Ordoñez et al. ${ }^{60}$ For Gen there is a band between 3500 to $3250 \mathrm{~cm}^{-1}$ which corresponds to $\mathrm{O}-\mathrm{H}$ stretching. In this interval, it is superposed to the band corresponding to $\mathrm{N}-\mathrm{H}$ stretching. The bands at 1054 and $1112 \mathrm{~cm}^{-1}$ correspond to the stretching of $\mathrm{C}-\mathrm{O}$ and $\mathrm{C}-\mathrm{O}-\mathrm{C}$ absorption, respectively. Similar results are reported for streptomycin, which has similar vibrational modes to Gen. ${ }^{63} \mathrm{~A}$ few bands are observed for Amox corresponding to $\mathrm{O}-\mathrm{H}$ and $\mathrm{N}-\mathrm{H}$ axial deformation in the region of 3532 and $3450 \mathrm{~cm}^{-1}$, respectively. The $\mathrm{C}=\mathrm{O}$ stretching $\beta$-lactam ring is quite 
high due to the change in Amox molecular dipole moment produced by this mode of vibration ${ }^{64,65}$ at $1776 \mathrm{~cm}^{-1}$. The absorption at $1686 \mathrm{~cm}^{-1}$ is attributed to the $\mathrm{C}=\mathrm{O}$ carbonyl group attached to the amide group. At $1483 \mathrm{~cm}^{-1}$ there is an overlay attributed to $\mathrm{N}-\mathrm{C}$ stretch and angular deformation for the amide group. The FTIR spectra for ZnBDC and ZIF-8 in the presence of Gen do not exhibit significant changes during the period of 1,3 and 7 days. On the other hand, both frameworks exhibit remarkable changes after suspension in the Amox solution. The FTIR spectrum for $\mathrm{ZnBDC}$ in the presence of Amox presents changes in chemical bonds. The characteristic vibrational modes related to asymmetric and symmetric $\mathrm{C}=\mathrm{O}$ stretching in the ZnBDC framework occur at 1576 and $1366 \mathrm{~cm}^{-1}$, respectively (Figure S13, SI section). ${ }^{43}$ In the presence of Amox, the two main vibrational peaks (1576 and $1145 \mathrm{~cm}^{-1}$ ) seen in the FTIR spectrum of ZIF-8 are no longer observed (Figure S16, SI section). The main peaks of the spectra for all samples is presented in Table S2 (SI section). It is most likely that these peaks underwent a shift and superposition with the bands associated with the Amox spectrum. The characteristic peak corresponding to $\mathrm{C}=\mathrm{O}$ stretching in the $\beta$-lactam ring of Amox at $1776 \mathrm{~cm}^{-1}$ is no longer observed in these spectra. The bands corresponding to the $\mathrm{N}-\mathrm{H}$ stretching, amide $\mathrm{C}=\mathrm{O}$, carbonyl groups and aromatic rings in the interval 3500 to $2600 \mathrm{~cm}^{-1}$ and to the $\mathrm{C}=\mathrm{C}$ stretching at $1615 \mathrm{~cm}^{-1}$ can still be observed in the FTIR spectra. The intensity decrease or disappearance of these peaks indicates that Amox may undergo degradation in the presence of the frameworks (see discussion of the MS data).

\section{UV-Vis spectroscopy}

Absorption spectra in the UV-Vis region were obtained to investigate if Amox underwent degradation upon contact with the frameworks. The UV-Vis spectra were measured for the pure Amox as well as its mixtures with ZnBDC or ZIF-8 in the period of 1, 2 and 3 days (Figures 2 and 3). The absorption spectrum for the pure Amox solution exhibits an electronic absorption band around 230 and $272 \mathrm{~nm} .{ }^{66,67}$ The band at $272 \mathrm{~nm}$ was used as reference to assure the structural conservation of Amox due to the higher sensitivity. Upon contact with the ZnBDC and ZIF-8 frameworks, the absorption band shifts ca. $80 \mathrm{~nm}$, i.e., from 272 to $350 \mathrm{~nm}$. The intensity ratio between the reference $(272 \mathrm{~nm})$ and the new band $(350 \mathrm{~nm})$ is significantly higher for ZIF-8 than for ZnBDC. The new adsorption band appears after 1 and 3 days in the presence of ZnBDC and ZIF-8, respectively. Therefore, the UV-Vis spectra show that Amox undergoes chemical degradation upon adsorption to the two $\mathrm{Zn}$-containing frameworks.

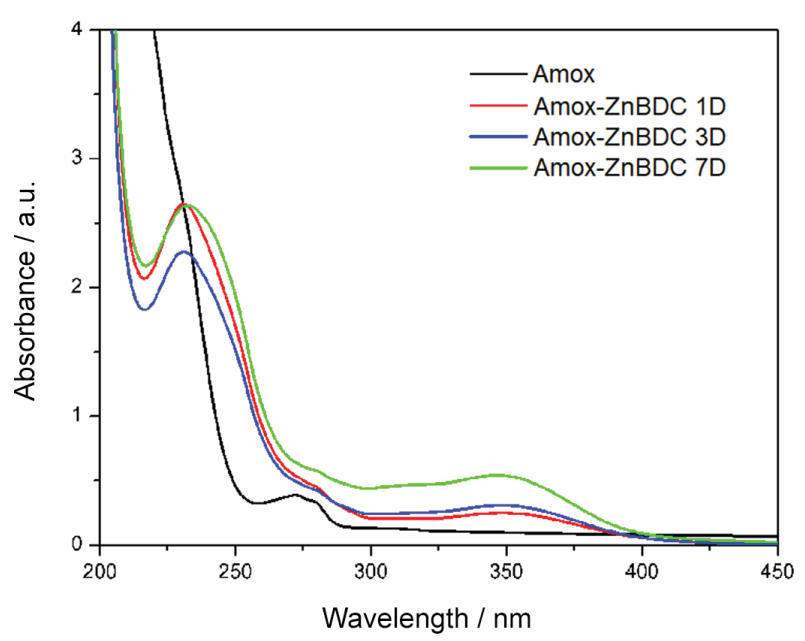

Figure 2. UV-Vis spectra of pure Amox and mixed with ZnBDC for 1, 3 and 7 days.

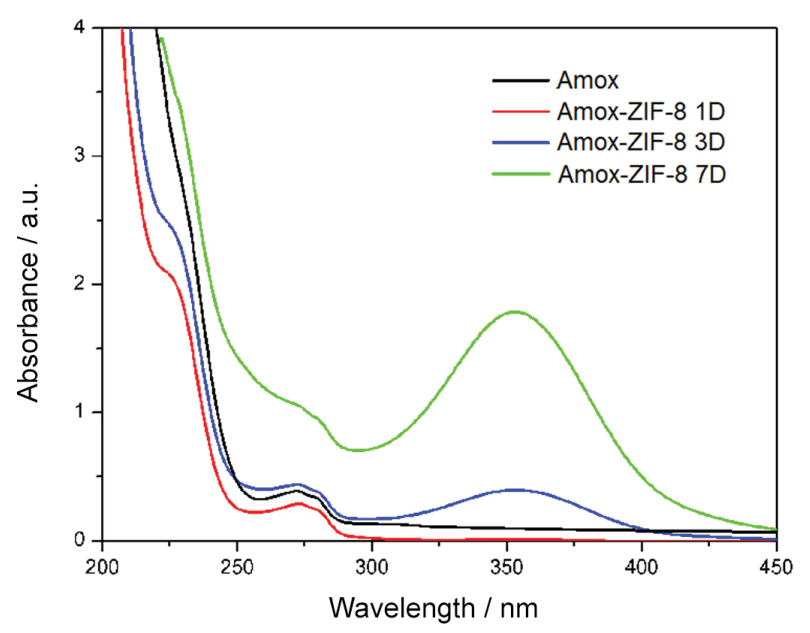

Figure 3. UV-Vis spectra of pure Amox and mixed with ZIF-8 for 1, 3 and 7 days.

\section{Mass spectrometry}

MS was used to detect the occurrence of degradation and detection of the resulting products in the supernatant and in the resulting solid after contact between frameworks and drugs.

The Amox MS revealed the presence of a peak corresponding to Amox at $\mathrm{m} / \mathrm{z} 366$ and its isotope at $\mathrm{m} / \mathrm{z} 367$ (Figure 4). A potassium adduct at $\mathrm{m} / \mathrm{z}, 404$ and a fragment generated by the loss of ammonia at $\mathrm{m} / \mathrm{z} 349$ were also observed. ${ }^{68}$ These assignments are consistent with MS measurements from Nägele and Moritz. ${ }^{69}$ The Amox dimer at $m / z 731$ with its respective isotope at $m / z, 732$, and potassium adduct of these two peaks at $\mathrm{m} / \mathrm{z} 769$ and 770 can also be observed in this spectrum. Similar behavior has been previously reported in literature. ${ }^{70}$

The MS for the solid phase of the sample containing Amox and frameworks shows the characteristic peak of 
Amox at $m / z 366$ (Figures 4 and 5). This peak is visible during the first 3 days of evaluation, disappearing after 7 days. The peak at $\mathrm{m} / \mathrm{z} 384$ is attributed to penicilloic acid, and is present at 3 and 7 days (Figures 4 and 5). ${ }^{70,71}$
The penicilloic acid is the degradation product formed by the opening of the $\beta$-lactam ring. ${ }^{70,71}$ The peak at $\mathrm{m} / \mathrm{z} 340$ is attributed to the decarboxilation of the penicilloic acid, denominated peniloic acid. ${ }^{69,72}$ The same Amox degradation

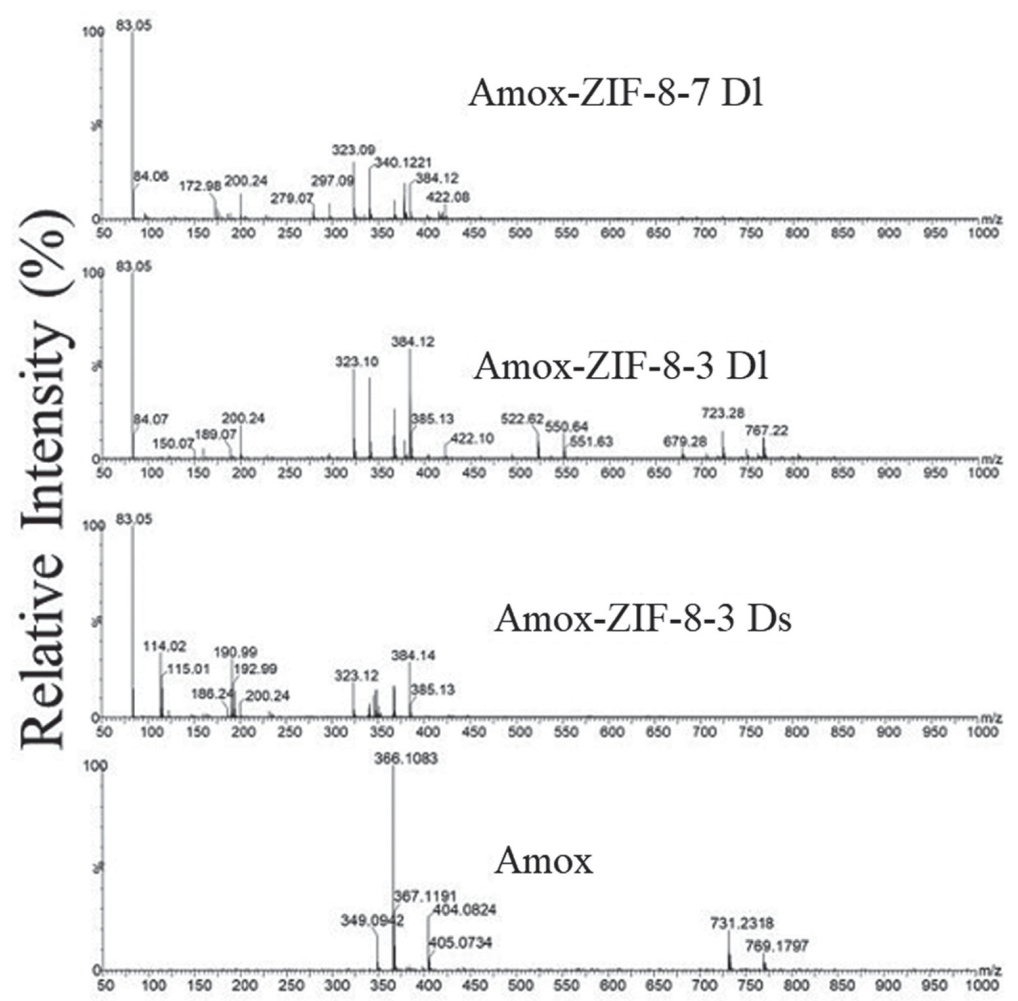

Figure 4. ESI(+)-MS of Amox and Amox-ZIF-8 (solid phase (Ds) and liquid (Dl)) at 3 and 7 days.

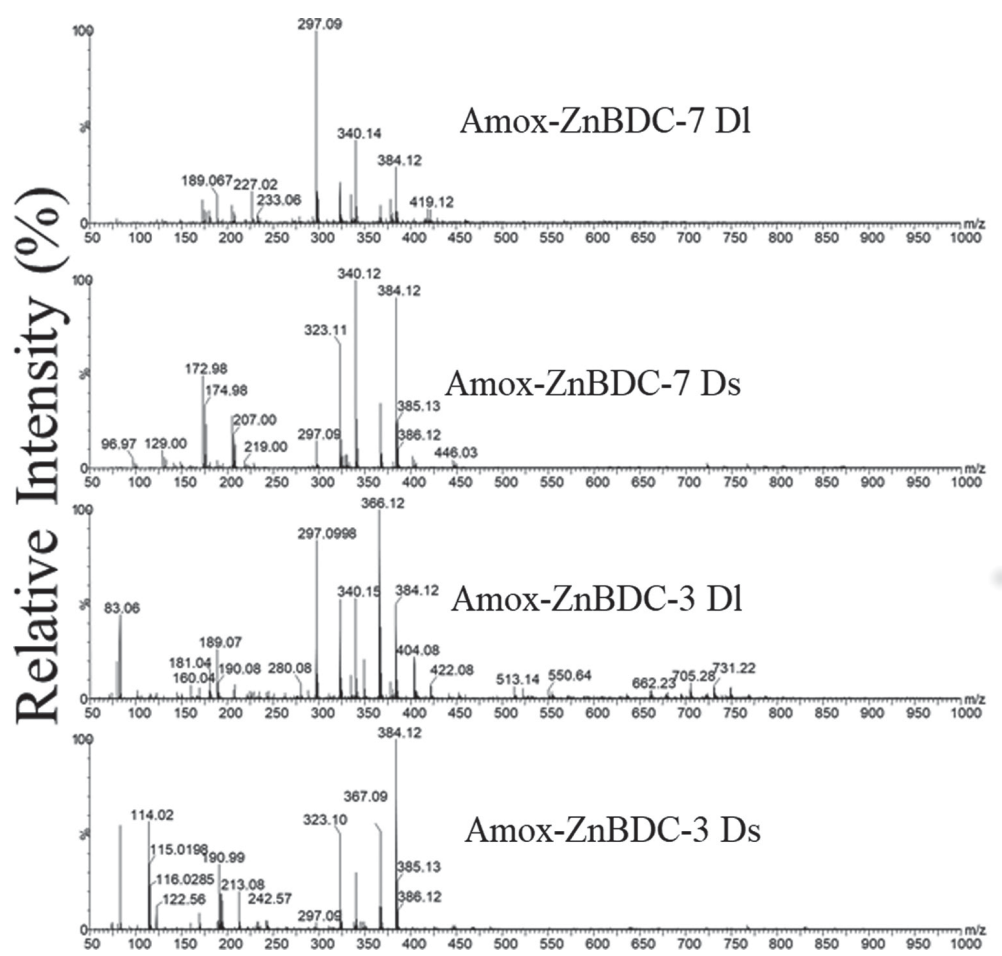

Figure 5. ESI(+)-MS of Amox and Amox-ZnBDC (solid phase (Ds) and liquid (Dl)) at 3 and 7 days. 
(a)
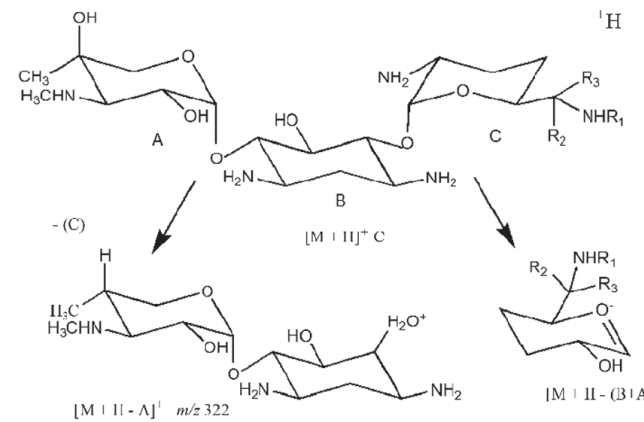

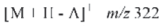

$|\mathrm{M}| \mathrm{II}-(\mathrm{B} \mid \mathrm{A}) \mid$

(b)

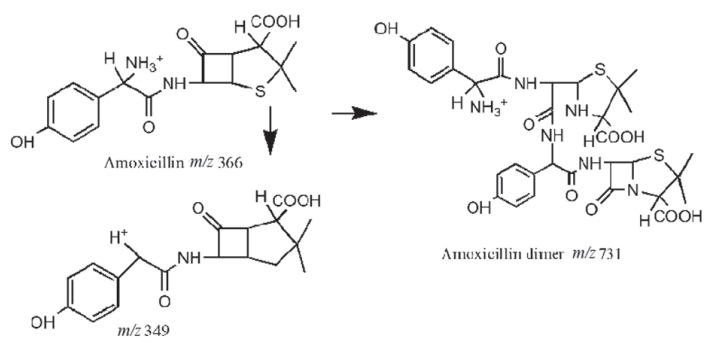

(c)

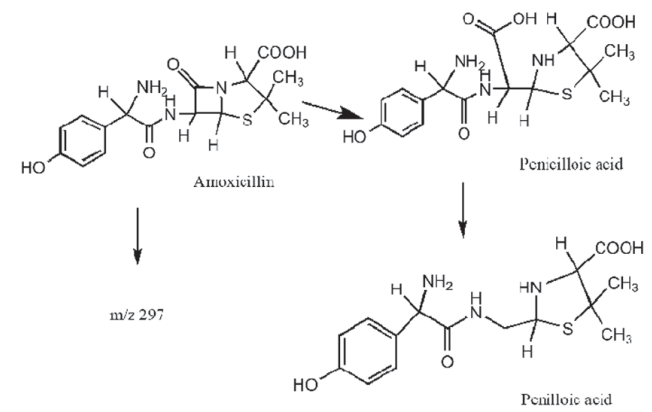

Figure 6. Fragmentation of (a) Gen and (b) Amox, and (c) degradation products of Amox.

products observed for the solid phase of the samples of the frameworks are observed for the supernatant (Figure 6). The MS of the solid and supernatant samples exhibit a peak at $m / z$ 297, which has not been previously identified as a degradation product of Amox in the absence of the frameworks (Figures 4 and 5). Its intensity increases with time, but the chemical structure of the compound associated with the peak at $m / z 297$ could not be determined at this point.

The MS data indicates that Gen does not undergo any chemical transformation in the presence of either ZIF-8 or ZnBDC (Figure 6). The MS for Gen do not exhibit any significant modification for the samples containing only Gen or Gen in the presence of the frameworks (whether ZIF-8 or ZnBDC). The MS measurements for the solid phase of the samples containing only the frameworks do not show any indication of fragmentation of Gen, suggesting that this drug does not adsorb to these frameworks (Figures S17 and S18, SI section). MS measurements for ZnBDC and ZIF-8 are presented in Figure S19 (SI section).

\section{Conclusions}

The findings reported here are consistent with the degradation of Amox by both ZnBDC and ZIF-8 frameworks. The degradation of Amox upon contact with the two MOFs is attributed to the presence of zinc ions, which is the catalytic agent in mono- and binuclear metallo- $\beta$-lactamases. ${ }^{73}$ Indeed, $\mathrm{Zn}^{2+}$ in this important class of enzymes is often tetracoordinated with imidazolate rings from histidine residues acting as ligands. These results are consistent with previous studies reporting on the role of $\mathrm{Zn}^{2+}$-containing materials in the degradation of antibiotics (Amox, ampicillin, penicillin-G, and penicillin-V) in aqueous solution and natural light exposure. ${ }^{74}$ These results evidence MOFs ZnBDC and ZIF-8 as new materials for Amox degradation. The products formed after degradation by the $\mathrm{Zn}$-containing frameworks were penicilloic and penilloic acids, which are non-toxic.

\section{Supplementary Information}

Supplementary information is available free of charge at http://jbcs.sbq.org.br as PDF file.

\section{Acknowledgments}

The Brazilian funding agencies CNPq, CAPES and FACEPE are acknowledged for providing financial support under grants Pronex APQ-0675-1.06/14, INCTNANOMARCS, Pronem APQ-0732-1.06/14 and BioMol/ CAPES (BioComp 23038.004630/2014-35).

\section{References}

1. Batt, A. L.; Kim, S.; Aga, D. S.; Chemosphere 2007, 68, 428.

2. Brown, K. D.; Kulis, J.; Thomson, B.; Chapman, T. H.; Mawhinney, D. B.; Sci. Total Environ. 2006, 366, 772.

3. Karthikeyan, K. G.; Meyer, M. T.; Sci. Total Environ. 2006, 361, 196.

4. Manzetti, S.; Ghisi, R.; Mar. Pollut. Bull. 2014, 79, 7.

5. Renew, J. E.; Huang, C.; J. Chromatogr. A 2004, 1042, 113.

6. Watkinson, A. J.; Murby, E. J.; Kolpin, D. W.; Costanzo, S. D.; Sci. Total Environ. 2009, 407, 2711.

7. Wise, R.; J. Antimicrob. Chemother. 2002, 49, 585.

8. Rizzo, L.; Manaia, C.; Merlin, C.; Schwartz, T.; Dagot, C.; Ploy, M. C.; Michael, I.; Fatta-Kassinos, D.; Sci. Total Environ. 2013, 447, 345 .

9. Andersson, D. I.; Hughes, D.; Nat. Rev. Microbiol. 2014, 12, 465.

10. Gozlan, I.; Rotstein, A.; Avisar, D.; Chemosphere 2013, 91, 985. 
11. Baquero, F.; Martínez, J. L.; Cantón, R.; Curr. Opin. Biotechnol. 2008, 19, 260.

12. Fram, M. S.; Belitz, K.; Sci. Total Environ. 2011, 409, 3409.

13. Jiang, L.; Hu, X.; Xu, T.; Zhang, H.; Sheng, D.; Yin, D.; Sci. Total Environ. 2013, 458-460, 267.

14. Khan, G. A.; Berglund, B.; Khan, K. M.; Lindgren, P. E.; Fick, J.; PLoS One 2013, 8, 4.

15. Kümmerer, K.; Chemosphere 2009, 75, 417.

16. Tuc, D. Q.; Elodie, M. G.; Pierre, L.; Fabrice, A.; Marie-Jeanne, T.; Martine, B.; Joelle, E.; Marc, C.; Sci. Total Environ. 2016, 575,758

17. García-Galán, M. J.; Díaz-Cruz, M. S.; Barceló, D.; TrAC, Trends Anal. Chem. 2008, 27, 1008.

18. Kennedy, K. A.; Rockwell, S.; Sartorelli, A. C.; Cancer Res. 1980, 40, 2356.

19. Choi, K. J.; Kim, S. G.; Kim, S. H.; J. Hazard. Mater. 2008 , $151,38$.

20. Putra, E. K.; Pranowo, R.; Sunarso, J.; Indraswati, N.; Ismadji, S.; Water Res. 2009, 43, 2419.

21. Zha, S.; Cheng, Y.; Gao, Y.; Chen, Z.; Megharaj, M.; Naidu, R.; Chem. Eng. J. 2014, 255, 141.

22. Elmolla, E. S.; Chaudhuri, M.; J. Hazard. Mater. 2009, 172, 1476.

23. Klauson, D.; Babkina, J.; Stepanova, K.; Krichevskaya, M.; Preis, S.; Catal. Today 2010, 151, 39.

24. Dimitrakopoulou, D.; Rethemiotaki, I.; Frontistis, Z.; Xekoukoulotakis, N. P.; Venieri, D.; Mantzavinos, D.; J. Environ. Manage. 2012, 98, 168.

25. Weng, X.; Sun, Q.; Lin, S.; Chen, Z.; Megharaj, M.; Naidu, R.; Chemosphere 2014, 103, 80.

26. Guo, W.; Wu, Q.-L.; Zhou, X.-J.; Cao, H.-O.; Du, J.-S.; Yin, R.-L.; Ren, N.-Q.; RSC Adv. 2015, 5, 52695.

27. Dong, H.; Zeng, G.; Tang, L.; Fan, C.; Zhang, C.; He, X.; He, Y.; Water Res. 2015, 79, 128.

28. Rodrigues, M. O.; de Paula, M. V.; Wanderley, K. A.; Vasconcelos, I. B.; Alves, S.; Soares, T. A.; Int. J. Quantum Chem. 2012, 112, 3346.

29. Wang, H.; Yuan, X.; Wu, Y.; Zeng, G.; Chen, X.; Leng, L.; Li, H.; Appl. Catal., B 2015, 174-175, 445.

30. Wang, C.; Liu, X.; Demir, N. K.; Chen, J. P.; Li, K.; Chem. Soc. Rev. 2016, 45, 5107.

31. Wang, K.; Huang, H.; Xue, W.; Liu, D.; Zhao, X.; Yuanlong, X.; Li, Z.; Qingyuan, Y.; Wang, L.; Zhong, C.; CrystEngComm 2015, 17, 3586.

32. Dias, E. M.; Petit, C.; J. Mater. Chem. A 2015, 3, 22484.

33. Gozlan, I.; Rotstein, A.; Avisar, D.; Environ. Chem. 2010, 7, 435.

34. Locatelli, M. A. F.; Sodré, F. F.; Jardim, W. F.; Arch. Environ. Contam. Toxicol. 2011, 60, 385.

35. Pérez-Parada, A.; Agüera, A.; Gómez-Ramos, M. D. M.; García-Reyes, J. F.; Heinzen, H.; Fernández-Alba, A. R.; Rapid Commun. Mass Spectrom. 2011, 25, 731.
36. Gómez-Ramos, M. D. M.; Pérez-Parada, A.; García-Reyes, J. F.; Fernández-Alba, A. R.; Agüera, A.; J. Chromatogr. A 2011, 1218, 8002.

37. Yang, C.; You, X.; Cheng, J.; Zheng, H.; Chen, Y.; Appl. Catal., B 2017, 200, 673.

38. Abazari, R.; Mahjoub, A. R.; Ultrason. Sonochem. 2018, 42, 577.

39. Abazari, R.; Reza Mahjoub, A.; Slawin, A. M. Z.; CarpenterWarren, C. L.; Ultrason. Sonochem. 2018, 42, 594.

40. Li, Q.; Zhang, W.; Miljanić, O. Š.; Sue, C. H.; Zhao, Y. L.; Liu, L.; Knobler, C. B.; Stoddart, J. F.; Yaghi, O. M.; Science 2009, 325,855 .

41. Lewis, K.; Nat. Rev. Drug Discovery 2013, 12, 371.

42. Wanderley, K. A.; Alves Jr., S.; Paiva-Santos, C. O.; Quim. Nova 2011, 34, 434.

43. Zhu, L. N.; Zhang, L. Z.; Wang, W. Z.; Liao, D. Z.; Cheng, P.; Jiang, Z. H.; Yan, S. P.; Inorg. Chem. Commun. 2002, 5, 1017.

44. Horcajada, P.; Serre, C.; Maurin, G.; Ramsahye, N. A; Balas, F.; Vallet-Regí, M.; Sebban, M.; Taulelle, F.; Férey, G.; J. Am. Chem. Soc. 2008, 130, 6774.

45. Goodford, P. J.; J. Med. Chem. 1985, $28,849$.

46. Morris, G. M.; Goodsell, D. S.; Halliday, R. S.; Huey, R.; Hart, W. E.; Belew, R. K.; Olson, A. J.; J. Comput. Chem. 1998, 19, 1639 .

47. Morris, G. M.; Huey, R.; Lindstrom, W.; Sanner, M. F.; Belew, R. K.; Goodsell, D. S.; Olson, A. J.; J. Comput. Chem. 2009, 30, 2785.

48. Huey, R.; Morris, G. M.; Olson, A. J.; Goodsell, D. S.; J. Comput. Chem. 2007, 28, 1145.

49. Sanner, M. F.; J. Mol. Graphics Modell. 1999, 17, 57.

50. Weiner, S. J.; Kollman, P. A.; Nguyen, D. T.; Case, D. A.; J. Comput. Chem. 1986, 7, 230.

51. Gasteiger, J.; Marsili, M.; Tetrahedron 1980, 36, 3219.

52. Soares, T. A.; Goodsell, D. S.; Ferreira, R.; Olson, A. J.; Briggs, J. M.; J. Mol. Recognit. 2000, 13, 146.

53. Soares, T. A.; Goodsell, D. S.; Briggs, J. M.; Ferreira, R.; Olson, A. J.; Biopolymers 1999, 50, 319.

54. Humphrey, W.; Dalke, A.; Schulten, K.; J. Mol. Graphics 1996, $14,33$.

55. MarvinSketch 5.3.1; ChemAxon, 2014. Available at http://www. chemaxon.com, accessed in April 2018.

56. Soares, T. A.; Lins, R. D.; Straatsma, T. P.; Briggs, J. M.; Biopolymers 2002, 65, 313.

57. Vasconcelos, I. B.; Silva, T. G.; Militão, G. C. G.; Soares, T. A.; Rodrigues, N. M.; Rodrigues, M. O.; Costa Jr., N. B.; Freire, R. O.; Junior, S. A.; RSC Adv. 2012, 2, 9437.

58. Park, K. S.; Ni, Z.; Côté, A. P.; Choi, J. Y.; Huang, R.; UribeRomo, F. J.; Chae, H. K.; O’Keeffe, M.; Yaghi, O. M.; Proc. Natl. Acad. Sci. U. S. A. 2006, 103, 10186.

59. Pan, Y.; Liu, Y.; Zeng, G.; Zhao, L.; Lai, Z.; Chem. Commun. 2011, 47, 2071. 
60. Ordoñez, M. J. C.; Balkus, K. J.; Ferraris, J. P.; Musselman, I. H.; J. Membr. Sci. 2010, 361, 28.

61. Montes, A.; Tenorio, A.; Gordillo, M. D.; Pereyra, C.; Martínez de la Ossa, E. J.; J. Supercrit. Fluids 2010, 51, 399.

62. Feng, S.; Shan, N.; Carpenter, K. J.; Org. Process Res. Dev. 2006, 10, 1212.

63. Nurkeeva, Z. S.; Khutoryanskiy, V. V.; Mun, G. A.; Sherbakova, M. V.; Ivaschenko, A. T.; Aitkhozhina, N. A.; Eur. J. Pharm. Biopharm. 2004, 57, 245.

64. Swaminathan, J.; Ramalingam, M.; Sethuraman, V.; Sundaraganesan, N.; Sebastian, S.; Kurt, M.; Spectrochim. Acta, Part A 2010, 75, 183.

65. Bebu, A.; Szabó, L.; Leopold, N.; Berindean, C.; David, L.; J. Mol. Struct. 2011, 993, 52.

66. Vallet-Regí, M.; Doadrio, J. C.; Doadrio, A. L.; Izquierdo-Barba, I.; Pérez-Pariente, J.; Solid State Ionics 2004, 172, 435.

67. Andreozzi, R.; Canterino, M.; Marotta, R.; Paxeus, N.; J. Hazard. Mater. 2005, 122, 243.
68. Lu, C. Y.; Feng, C. H.; J. Sep. Sci. 2007, 30, 329.

69. Nägele, E.; Moritz, R.; J. Am. Soc. Mass Spectrom. 2005, 16, 1670.

70. Valvo, L.; Ciranni, E.; Alimenti, R.; Alimonti, S.; Draisci, R.; Giannetti, L.; Lucentini, L.; J. Chromatogr. A 1998, 797, 311.

71. Magureanu, M.; Piroi, D.; Mandache, N. B.; David, V.; Medvedovici, A; Bradu, C.; Parvulescu, V. I.; Water Res. 2011, 45, 3407.

72. Trovó, A. G.; Pupo Nogueira, R. F.; Agüera, A.; Fernandez-Alba, A. R.; Malato, S.; Water Res. 2011, 45, 1394.

73. Tamilselvi, A.; Mugesh, G.; J. Biol. Inorg. Chem. 2008, 13, 1039.

74. Navarro, P. G.; Hernández Blázquez, I.; Quintero Osso, B.; Martínez De Las Parras, P. J.; Martínez Puentedura, M. I.; Márquez García, A. A.; Int. J. Biol. Macromol. 2003, 33, 159.

Submitted: January 29, 2018 Published online: April 26, 2018 